\title{
Respiratory mechanics assessed by pressure-volume loops with forced oscillations
}

\author{
C. M. IONESCU and R. M. C. DE KEYSER
}

Department of Electrical Energy, Systems and Automation, Ghent University, Ghent, Belgium

\section{Introduction}

Forced Oscillation Technique (FOT) is an efficient and easy-to-apply non-invasive lung function test (Pride 1996) which is not yet used at its full potential. The standard FOT test consists in superimposing an oscilatory pressure on the spontaneous breathing of the subject (in this case a $5 \mathrm{~Hz}$ sinusoidal pressure signal). This abstract presents a complementary indicator of respiratory mechanics, i.e. the dynamic pressure-volume (PV) loop, obtained via the FOT test. Although pressure and flow are achieved by applying the FOT test, the volume can be easily obtained by simply integrating the flow signal. Here, a preliminary result indicates differences that can be obtained by use of PV-loops between a person with normal respiration mechanics and a person with increased airway resistance.

\section{PV loops}

PV loops plot pressure versus volume during inspiration and expiration. Pressure volume loops can be generated under static (no flow) conditions or under dynamic conditions (with flow during a breath). They also can be generated during forced breathing or normal breathing of the patient. In this abstract, results are presented during normal breathing of the patient and using the non-invasive lung function test (FOT). Figure 1 depicts PV loops generated during normal breathing, whereas the frequencies above $2 \mathrm{~Hz}$ are filtered out (eliminating thus the sinusoidal oscillation in the loop's curves). The lung is inflated and the airway pressure increases throughout inspiration. This pressure will depend on the compliance of the respiratory system, the resistive properties and the inspiratory flow rate (Navajas and Farré 1999).

\section{Results and discussion}

Respiratory failure is generally any condition that affects breathing function or the lungs themselves and can result in failure of the lungs to function properly. The dynamic pressure-volume (PV) characteristics are able to underline some of the mechanical properties of the subjects, and therefore to provide initial diagnosis. In this contribution, the PV loops of two subjects have been compared. The subjects are about 24 years old, male, one of them (dashed line) presenting difficulties in breathing (increased airways resistance). From figure 1 can be distinguished between these two subjects.

From the dynamic PV loops, the compliance can be easily obtained as $C=\Delta V / \Delta P$. The change in steepness of the inspiratory branch of the PV loop is proportional to the change in lung compliance. In this case, there is no significant difference in compliance between the two cases. On the other hand, if the resistance changes, this will not affect the steepness of the branch, but it will change position (A). Further on, if the PV loop starts to become flatter in the upper part of the inspiratory branch, this could be an indication of over-extension of certain areas of the lung. This aspect is not experienced in this case.

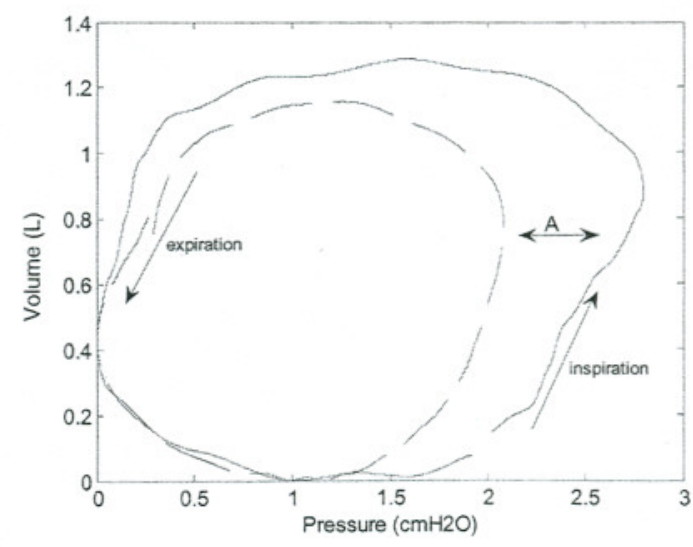

Figure 1. Dynamic PV loops obtained for a healthy patient (continuous line) and for a patient with increased airway resistance (dashed line). 


\section{Conclusion}

When the natural ventilation of the patient does not provide the optimal breathing conditions, artificial ventilation support is provided. This is done by applying either a constant pressure or constant flow, and PV loops are obtained to measure the efficiency of this setup. The abnormal performances in terms of ventilation of the lung function can be determined from PV loops. In this contribution, the PV loops during normal breathing conditions have been depicted, from a simple, noninvasive forced oscillations test. The PV loops were compared in the case of resistance changes. Such additional information could be then used to validate and complete the diagnosis procedure (Ionescu and De Keyser 2004).

\section{References}

C. Ionescu and R. De Keyser, "Towards a novel non-invasive diagnosis method for the human respiratory system", Proc. IEEE X Medit. Conf. on Med. and Biol. Eng. Ischia, Naples, Italy, 4p, 2004.

D. Navajas and R Farré, "Oscillation mechanics", Eur. Resp. Monograph, 12, pp. $112-140,1999$.

N.B. Pride, Lung Function Tests-Physiological Principles and Clinical Applications, J.M.B. Hughes and N.B. Pride, Eds., London: W.B. Saunders, 1996, pp. 3-72. 